\title{
Advances in the management of macular degeneration
}

\section{Michael Singer}

Address: Medical Center Ophthalmology Associates, San Antonio, Texas, USA

Email: msinger1@earthlink.net

Fl000Prime Reports 2014, 6:29 (doi:10.12703/P6-29)

All FI000Prime Reports articles are distributed under the terms of the Creative Commons Attribution-Non Commercial License (http://creativecommons.org/licenses/by-nc/3.0/legalcode), which permits non-commercial use, distribution, and reproduction in any medium, provided the original work is properly cited.

The electronic version of this article is the complete one and can be found at: http://f1000.com/prime/reports/m/6/29

\begin{abstract}
Current management of age-related macular degeneration (AMD) can be divided into two categories: first, anti-vasoendothelial growth factor (anti-VEGF) injection for wet macular degeneration; second, anti-oxidant vitamins for dry macular degeneration. New therapies are being developed for both of these diseases using novel technologies and different modes of administration. The hope is that some of these therapies will achieve significant improvement to current management and prevent future loss of vision in this devastating eye condition.
\end{abstract}

\section{Introduction}

AMD remains the leading cause of irreversible blindness in the industrialized world. It affects 30-50 million people worldwide. Of these, 1.5 million people have wet macular degeneration with 600,000 new cases diagnosed per year [1].

This disease can be divided into two basic subtypes. Wet or neovascular AMD (10\% of cases) is the growth of new blood vessels from the choroid under the macular region, leading to rapid blindness. The other type, dry AMD $(90 \%$ of cases), is the much more insidious subtype, in which vision slowly decreases over many years due to loss of photoreceptors and development of geographic atrophy.

\section{Recent advances}

Over the last decade, numerous therapeutic options have become available for neovascular AMD, most notably anti-VEGF medications. Examples of these are ranibizumab (Lucnetis, Genentech), bevacizumab (Avastin, Genentech) and aflibercept (Eylea, Regeneron). Clinical studies, such as Anchor and Marina (ranibizumab), View 1 and 2 (aflibercept) and CATT and IVAN (bevacizumab), have proven that these medicines are effective in achieving stabilization in the vast majority of cases and increasing vision in a substantial minority of cases. While these drugs are far more effective than previously available options (with $90 \%$ of patients maintaining current vision and $40 \%$ gaining vision) there still remains room for improvement [2-5].

In order to be effective, anti-VEGF medications must be used relatively early in the disease process, before scar formation has occurred. These medications have the potential to increase the risk of thromboembolic events in an already susceptible population. Intravitreal injections, regardless of the drug used, have well documented risks including bacterial endophthalmitis, sterile endophthalmitis, cataract formation, hemorrhage, and retinal detachment.

In addition, these medications may become less effective with repeated use, a phenomenon known as tacchyphylaxis. Furthermore, the widespread adaptation of these drugs has placed an increased strain on medical practices, both in terms of cash flow management and inventory control. Logistical difficulties exist for providers and patients alike with frequent office visits for testing, monitoring and treatment. In addition, both CATT and IVAN clinical studies have shown that multiple injections can lead to retinal thinning and geographic attrophy $[5,6]$.

So what does the future hold for treatment of wet macular degeneration? 
In the short term, two new compounds have the potential for being therapeutic options. Fovista ${ }^{\mathrm{TM}}$ is a product by Ophthotech currently in phase 3 clinical trials. Delivered by intravitreal injection, Fovista ${ }^{\mathrm{TM}}$ prevents plateletderived growth factor from binding to its natural receptor on pericytes, thus causing pericytes to be stripped from newly formed abnormal blood vessels. Left unprotected, the endothelial cells are highly vulnerable to the effects of anti-VEGF drugs. Because of this unique action of being able to strip pericytes, the drug may work on both immature and, slightly later in the disease process, more mature vessels. A phase 2 study demonstrated that patients receiving the combination of $1.5 \mathrm{mg}$ of Fovista ${ }^{\mathrm{TM}}$ and ranibizumab (Lucentis ${ }^{\circledR}$ ) gained a mean of 10.6 letters from baseline on a standardized chart of vision testing, compared to a mean gain of 6.5 letters from baseline for patients receiving ranibizumab monotherapy. This represents a $62 \%$ comparative benefit from baseline $[7,8]$.

DARPin (designed ankyrin repeat proteins) is another medicine in clinical trial. DARPins are genetically engineered antibody mimetic proteins, and typically exhibit highly specific and high-affinity target protein binding. They are derived from natural ankyrin proteins and are composed of a minimum of three, but usually four or five, repeat motifs of ankyrin proteins. Initially developed by molecular partners, this molecule targets VEGF A using different technology than bevacizumab, aflibercept, and ranibizumab. MP0112, the first therapeutic DARPin candidate, is targeted for decreasing VEGF (NCT01086761). The molecule (purchased by Allergan) had a phase 2 study that suggested that DARPin has the potential to achieve a longer dosing interval than that observed with ranibizumab. Ongoing studies are exploring the potential benefits of loading doses [9]. In the longer term, there are a number of different concepts that show promise.

Topical eye drops may potentially eliminate many of the risks associated with intravitreal injections. Ohr Pharmaceuticals is testing a version of squalamine lactate that works by intracellular inhibition of angiogenesis, and is currently in phase 2 studies as an eyedrop [10]. In preclinical studies, PanOptica is testing PAN-90806, a novel, small-molecule selective VEGF receptor antagonist that shows promise as a topical treatment for wet AMD and diabetic retinopathy [11].

A sustained delivery compound or device might potentially reduce the number of office visits and treatments. Alcon is in phase 1 trials of EBSA 1008, using a humanized singlechain antibody fragment, which is an extremely potent pan-VEGF-A inhibitor via a continued infusion solution.
If successful, this compound could lead to micro-volume treatment doses, which could be suitable for long term use (NCT01849692).

In terms of dry macular degeneration, the AREDS study is still the gold standard for demonstrating the value of vitamins in preventing the progression of dry macular degeneration. In 2013 the AREDS 2 study was performed, which tested adding carotenoid vitamins lutein and zeaxanthin, as well as omega 3, to the original AREDS formula. The original formula tested vitamins $\mathrm{E}$ and $\mathrm{C}$ as well as the carotenoid vitamin beta-carotene. The trial showed that patients who took the supplement over 5 years had a $25 \%$ less chance of progressing to advanced AMD than those who took placebo.

The AREDS 2 study results showed that adding lutein, zeaxanthin and omega 3 did not improve the original AREDS formula overall. When subgroup analysis was performed, there was some benefit to patients who did not get beta-carotene, and to those who were given lutein and zeaxanthin, when the patients' diets were deficient in these nutrients. The study also looked at the dosage of zinc and found no difference between low dose and high dose.

When comparing patients who took the ARED supplement containing lutein and zeaxanthin with those who took beta-carotene, there was an $18 \%$ reduction in the development of advanced AMD. In addition, the patients who took the ARED supplement containing lutein and zeaxanthin, and whose daily lutein and zeaxanthin was low, had 25\% less risk of developing advanced AMD.

The study also showed that patients who took the betacarotene version of the AREDS formula and who were former smokers had an increased risk of developing lung cancer [12].

In terms of dry macular degeneration, there are a number of medicines being investigated that utilize different mechanisms. These can be divided by their different mechanisms of action: (a) neuroprotection; (b) reduction of toxic byproducts; (c) other mechanisms; (d) visual cycle modulation; (e) suppression of inflammation; (f) stem cell replacement; (g) complement inhibition.

\section{Neuroprotection}

There are two neuroprotection molecules in the pipeline: ciliary neurotrophic factor (CNTF) and brimonidine tartrate.

Ciliary neurotrophic factor (Neurotech) is a neuroprotective cytokine that prevents photoreceptor degeneration. 
Encapsulated cell technology utilizes a delivery system that achieves a controlled, continuous, sustained delivery of CNTF. A randomized, double masked, sham-controlled multi-center phase 2 study was performed. There were three arms: high dose (24 patients), low dose (12 patients) and placebo (12 patients). The primary endpoint was vision at 12 months. The secondary endpoints were optical coherence tomography (OCT) macular thickness, volume by OCT and lesion size on fundus photography. The phase 2 results demonstrated visual acuity stabilization with $96 \%$ of the high dose patients achieving stabilization (losing less than 15 Early Treatment Diabetic Retinopathy study [EDTRS] letters) and retinal thickness preservation $v$ s. $75 \%$ of the sham group [13].

Brimonidine (Allergan) is an Alpha-2 adrenergic receptor agonist, which has been shown to be neuroprotective in animal models. There was a phase 2 study using a sustained delivery system similar to the dexmathesone intravitreal implant (Ozurdex) model. It is currently being reformulated and clinical trials are due to start in the future [14].

\section{Reduction of toxic byproducts}

Other medications work by reduction of toxic byproduct accumulations. Three examples of these medications are subcutaneous glatiramer acetate, intravenous RN6G (anti-amyloid $\beta$ antibody), and GSK 933776.

Glatiramer acetate (Copaxone) appears to reduce amyloid-induced retinal microglial cytotoxicity, allowing a neuroprotective phenotype of microglia to form. It is currently US Food and Drug Administration (FDA) approved for the treatment of multiple sclerosis. A phase 1 study using a subcutaneous injection (dose of $20 \mathrm{mg}$ ) of this medication has been completed and a phase 2 study is underway [15].

RN6G (PF-4382923 Pfizer) is a humanized monoclonal antibody against $\mathrm{A} \beta 40$ and $\mathrm{A} \beta 4 \mathrm{~B} 42$. It binds to amyloid that accumulates in drusen. RN6G binds and sequesters $\mathrm{A} \beta$ species (targets C-termini of $\mathrm{A} \beta 40$ and $\mathrm{A} \beta 42$ ) in the periphery, reducing the pool of toxic species in the retina (as described by Ding et al. in 2008 [16]). RN6G also prevents accumulation of $\mathrm{A} \beta 40$ and $\mathrm{A} \beta 42$ that are implicated in neurodegenerative disorders. It is given by intravenous administration. The phase 1 study is completed and the phase 2 is ongoing (NCT00877032, NCT01003691, NCT01577381, [17]).

GSK 933776 (Glaxo Smith Kline) is a humanized monoclonal antibody against amyloid-beta, which is given by an intravitreal injection into the eye. The phase 1 study has been completed in patients with advanced AMD and the phase 2 study evaluating 6 monthly doses on the growth of geographic atrophy is currently underway [17].

\section{Medicines that work by other mechanisms}

MC-1101 (Macuclear) is a topically administered eye drop. It works by increasing ocular blood flow in the choroidal blood vessels, thus preventing the rupture of Bruch membrane. A successful phase $1 \mathrm{~b} /$ proof of concept has been completed. A phase 2/3 vehicle-controlled, double-masked, single-center study is currently being conducted over the course of 2 years.

Another mechanism is an Aldehyde trap (Aldexa). Aldehydes are toxic mediators known to be elevated in patients with dry AMD. These medications bind to malondialdehyde, 4-hydroxynonenal, and retinaldehyde, three common toxic aldehydes. The effect is to lower excessive levels of these aldehydes in AMD patients, which represents an innovative strategy for potential treatment. It is currently in preclinical development [18].

Doxycycline (Oracea-Gladerma) is another medication currently in trials, which helps prevent photoreceptor loss in AMD. It is currently approved for treating the inflammatory lesions of rosacea. The TOGA trial is a multicenter phase $2 / 3$ study to assess the efficacy and safety of daily oral administration of doxycycline, compared with placebo, on the rate of change in the area of geographic atrophy (NCT01782989).

Sustained release steroid implants are being evaluated for dry AMD as well. Fluocinolone acetonide (Alimera), which is currently approved in Europe for diabetic macular edema, has a phase 2 study planned for geographic atrophy, in which two doses of 0.2 and $0.5 \mu \mathrm{g} /$ day fluocinolone acetonide are being tested for the treatment of dry AMD [19].

\section{Complement inhibition}

There are numerous steps throughout the complement cascade that medicines are targeting.

The following medicines target different stages in the complement pathway: LFG 316 (C5 inhibition); POT-4 (C3 inhibition); Eculizumab (C5 inhibition); Anti-Factor D.

LFG 316 (Novartis) is an antibody against the C5 portion of the complement pathway.

It is administered by intravitreal injection. The phase 1 study has been completed in patients with advanced 
AMD, geographic atrophy, or choroidal neovascularization (CNV).

The phase 2 study is currently ongoing, evaluating 6 monthly doses on the growth of geographic atrophy. A long-acting delivery system is in preclinical development [19].

POT-4, now known as AL-78898A (Alcon), is a cyclic peptide that irreversibly binds to $\mathrm{C} 3$ to inhibit complement pathways and prevent membrane attack complex formation. It forms a gel when injected into the vitreous. The phase 2 study evaluated 12 monthly doses on the progression of geographic atrophy. The results have not yet been released [15].

Eculizumab (Alexion) is the first FDA approved complement inhibitor for the treatment of paroxysmal nocturnal hemoglobinuria. It is a humanized IgG antibody against C5. It is currently administered by intravenous (systemic) infusion. It works by preventing lysis of red blood cells and the formation of membrane attack complex. The phase 2 study (COMPLETE) did not demonstrate decreased drusen volume or prevent geographic atrophy growth in two separate cohorts. A one year follow up is ongoing [15].

Anti-factor D Fab/lampalizumab (Genentech) is a humanized monoclonal antibody fragment targeting complement factor D. It is designed to inhibit complement activation and chronic inflammation in tissues. The phase $1 / 2$ clinical trial (MAHALO) evaluated anti-factor D for geographic atrophy associated with dry AMD. The complement inhibitor lampalizumab reduces disease progression by $20 \%$ and by $44 \%$ in a biomarker-defined subset, according to results from the MAHALO study. Of the biomarkers identified, patients with complement factor I showed a protective response to the medication in preventing disease progression. These data were presented at the 2013 American Academy of Ophthalmology subspecialty day meeting. A phase 3 study is planned [20].

\section{Visual cycle modulators}

Visual cycle modulators are another mechanism of action being studied to help prevent photoreceptor and retinal pigment epithelium (RPE) loss in AMD. Visual cycle modulation essentially "slows down" the activity of the rods and reduces the metabolic load on the cones. Rod cell deterioration is common with age. These medications reduce the accumulation of toxic fluorophors (A2E) and lipofuscin and, as a result, prevent photoreceptor/RPE cell loss. Two examples of these medications are oral fenretinide and oral ACU-4429. Fenretinide (Sirion) conducted a phase 2 study with 246 patients with geographic atrophy. The medication was an oral daily soft gel capsule given with the evening meal. Patients were randomized 1:1:1 to $100 \mathrm{mg}, 300 \mathrm{mg}$, and placebo. It was a 2-year study, with interim analysis after 1 year. The medication slowed geographic atrophy growth in $43 \%$ of patients, but this was only evident in a subgroup analysis, the results were not statistically significant, and no phase 3 study is planned [21].

Acucela has a small molecule, ACU-4429, which is given as an oral tablet. It is selective for rod photoreceptors and has demonstrated efficacy in multiple preclinical models, such as the aged animal model, the light damage model and the retinopathy of prematurity (ROP) model. The medication is designed to prevent or inhibit the generation of toxic byproducts of the visual cycle. It slows the rod visual cycle and is administered as a daily pill given as an evening dose.

The phase 2/3 trial SEATTLE is a randomized, doublemasked, placebo-controlled, multi-center safety and efficacy study in patients with dry AMD/geographic atrophy. There are three planned escalating dose levels of ACU-4429 (2.5, 5 and $10 \mathrm{mg}) v s$. placebo (10 mg will have dose escalation). The pill is given at night due to problems with night vision. The dosing is once-a-day over two years. The study, which is currently ongoing, is expected to enroll approximately 440 patients $[22,23]$.

\section{Stem cell replacement}

Another potential therapy is the delivery of stem cells. Two of the companies currently pursuing this therapy are Advanced Cell Technology (ACT), and Janssen Biotech (J\&J and I-science) (CNTO 2476).

ACT is using human embryonic stem cells (hESCs). There are two separate phase $1 / 2$ trials to test the safety of the hESC-derivedRPE cellular therapy for dry AMD and for Stargardt macular dystrophy. The first study is a single uniocular subretinal infusion of MA09-hRPE cells in 1 of 4 dose levelsNCT01345006). The other is a phase $1 / 2 \mathrm{a}$ study to evaluate the safety and tolerability of MA09-hRPE cellular therapy in patients with advanced dry AMD (NCT01344993).

CNTO 2476 uses stem cells derived from an umbilical source. The cells are delivered via subretinal injection, an external approach using iTrack microcatheter and an endoscope. There is a phase $1 / 2 \mathrm{a}$ study ongoing in geographic atrophy patients, and currently two centers are enrolling (NCT01226628).

\section{Future directions}

In the future, the goal in wet macular degeneration will be to completely arrest the disease via a sustained delivery 
system, utilizing a combination of medicines that have different mechanisms of action. In terms of dry AMD, the hope is to not only arrest the disease but to find a way to restore photoreceptor function. Although there is a long way to go, there are a number of very promising therapies on the horizon for both subtypes of this disease.

\section{Abbreviations}

ACT, Advanced Cell Technology; AMD, age-related macular degeneration; anti-VEGF, anti-vascular endothelial growth factor; CNTF, ciliary neurotrophic factor; DARPin, designed ankyrin repeat protein; hESC, human embryonic stem cell; RPE, Retinal pigment epithelium.

\section{Disclosures}

Michael Singer has been a research support consultant speaker for Allergan, Genentech and Thrombogenics, a research support speaker for Regeneron, a research support consultant for Acucela, a member of CNTO Independent safety monitoring committee, and research support at Alcon, Ophthotech and Ohr.

\section{References}

I. AMD Alliance International. [http://www.amdalliance.org/]

2. Brown DM, Kaiser PK, Michels M, Soubrane G, Heier JS, Kim RY, Sy JP, Schneider S: ANCHOR Study Group: Ranibizumab versus verteporfin for neovascular age-related macular degeneration. N Eng J Med 2006, 355: |432-44.

\section{FlOOOPrime

RECOMMENDED

3. Rosenfeld PJ, Brown DM, Heier JS, Boyer DS, Kaiser PK, Chung CY, Kim RY: MARINA Study Group. Ranibizumab for neovascular age related macular degeneration. N Engl J Med 2006, 355:|4-|43|.

\section{FIOOOPrime
RECOMMENDED}

4. Heier JS, Brown DM, Chong V, Korobelnik JF, Kaiser PK, Nguyen QD, Kirchhof B, Ho A, Ogura Y, Yancopoulos GD, Stahl N, Vitti R, Berliner AJ, Soo Y, Anderesi M, Groetzbach G, Sommerauer B, Sandbrink R, Simader C, Schmidt-Erfurth U: VIEW I and VIEW 2 Study Groups. Intravitreal aflibercept (VEGF trap-eye) in wet age-related macular degeneration. Ophthalmology 2012, I | 9:2537-48.

\section{FIOOOPrime}

\section{RECOMMENDED}

5. Comparison of Age-Related Macular Degeneration Treatments Trials (CATT) Research Group, Martin OF, Maguire MG: Ranibizumab and bevacizumab for treatment of neovascular age-related macular degeneration: Two-year results. Ophthalmology 2012, | | 9: | 388-98.

\section{FlOOOPrime}

6. Chakravarthy U, Harding SP, Rogers CA, Downes SM, Lotery AJ, Culliford LA, Reeves BC; IVAN study investigators: Alternative treatments to inhibit VEGF in age-related choroidal neovascularisation: 2-year findings of the IVAN randomised controlled trial. Lancet 20I3, 382: I258-67.

7. Ophthotech Corporation. [www.ophthotech.com]

8. Dugel, PU: Phase 2b clinical trial of Fovista"' anti-PDGF therapy $(1.5 \mathrm{mg})$ in patients with neovascular age-related macular degeneration (wet AMO) [abstract]. Presented at Retina Subspecialty Day at the American Academy of Ophthalmology Annual Meeting (AAO) I0-I3 Nov 2012, Chicago, IL.

9. Callanan, D: Designed Ankyrin Repeat Proteins (DARPins): Proof of Concept Study [abstract]. Presented at Retina Subspecialty Day at the American Academy of Ophthalmology Annual Meeting (AAO) I6-19 Nov 20I3, Louisiana, LA.

10. Ohr Pharmaceutical. [http://www.ohrpharmaceutical.com]

II. PanOptica, Inc. [http://www.panopticapharma.com]

12. Age-Related Eye Disease Study 2 Research Group: Lutein+ Zeaxanthin and Omega-3 Fatty Acids for Age- Related Macular Degeneration: The Age-Related Eye Disease 2 (AREDS) Randomized Clinical Trial. JAMA 20I3, 309:2005-I 5.

FlOOOPrime
RECOMMENDED

13. NT 50I program. [http://www.Neurotechusa.com]

14. Kupperman BD: Drug Delivery Implants for Geographic Atrophy. Retina today May 2012. [http://bmctoday.net/retinatoday/ 2012/05/article.asp?f=drug-delivery-implants-for-geographic-atrophy]

15. Roe R, Boyer D: The Pipeline for Dry macular Degeneration. Review of Ophthalmology 201 I. [http://www.revophth.com/content/d/ retinal_insider/i/l709/c/31319/]

16. Ding J, Lin J, Mace BE, Herrmann R, Sullivan P, Bowes Rickman C: Targeting age-related macular degeneration with Alzheimer's disease based immunotherapies: anti-amyloid-beta antibody attenuates pathologies in an age-related macular degeneration mouse model. Vision Res 2008, 48:339-45.

17. Evans JB, Syned BA: New hope for dry AMD? Nat Rev Drug Discov 20I3, I2:501-2.

18. Chioy G: Is Dry AMD Treatable? Retina Today May 2012. [http:// bmctoday.net/retinatoday/2012/05/article.asp?f=is-dry-amd-treatable]

19. Ambati J, Atkinson J, Gelfand B: Immunology of age realted macular degeneration. Nat Rev Immunol 2013, I3:438-5I.

20. Hetwick, C: MAHALO Finds New Biomarker for Dry Macular Degeneration, Medscape Medical News 21 Nov 2013. [http://www. medscape.com/viewarticle/8I4822]

21. Mata NL, Lichter JB, Vogel R, Han Y, Bui TV, Singerman LJ: Investigation of oral fenretinide for treatment of geographic atrophy in age-related macular degeneration. Retina 2013, 33:498-507.

22. Mata, NL, Kubota R, Schneider S, Kissner J, Birch DG, Dugel PU: Pharmacodynamics Of Visual Cycle Modulation In the Treatment of GA, Emerging data from clinical studies demonstrate the biological activity of emixustat in the retina. Retinal Physician 2003. [http://www.retinalphysician.com/articleviewer.aspx?articlelD = $10886 \mid]$

23. Kubota R, Boman NL, David R, Mallikaarjun S, Patil S, Birch D: Safety and effect on rod function of ACU-4429, a novel smallmolecule visual cycle modulator. Retina 20I2, 32: I83-8. 